\title{
Twinfocus, a concentrated photovoltaic module based on mature technologies
}

\author{
Piergiorgio Antonini ${ }^{1,2,3}$, Sandro Centro ${ }^{2,3}$, Stelvio Golfetto ${ }^{3}$ and Alessandro Saccà a,4,a $^{2, a}$ \\ ${ }^{1}$ Centro Fermi, P.zza del Viminale 1, 00184 Roma, Italy \\ ${ }^{2}$ Physics and Astronomy Department, University of Padova, via Marzolo 8, 35131 Padova, Italy \\ ${ }^{3}$ AtemEnergia srl, Gall. Borromeo 3, 35137 Padova, Italy \\ ${ }^{4}$ Department of Industrial Engineering, University of Padova, via Venezia 1, 35131 Padova, Italy
}

\begin{abstract}
Among solar power generation, concentrated photovoltaics (CPV) based on multijunction (MJ) solar cells, is one of the most promising technology for hot climates. The fact that multijunction solar cells based on direct band gap semiconductors demonstrate lower dependence on temperature than silicon solar cells boosted their use in concentrated photovoltaics modules. Departing from the mainstream design of Fresnel lenses, the CPV module based on TwinFocus design with off-axis quasi parabolic mirrors differentiates itself for its compactness and the possibility of easy integration also in roof-top applications. A detailed description of the module and of the systems will be given together with measured performances, and expectations for the next release.
\end{abstract}

\section{Introduction}

During the last few years the spin-off AtemEnergia designed and produced some prototypes of the TwinFocus concentrator. The system and the working team have already been introduced in [1] and [2] and here we recall that such spin-off was born from the collaboration of research institutions with industrial partners that operate in the injection moulding business. This synergy brought the group to the creation of a new CPV module, which optical primary element is based on the state of art of the injection moulding plastic techniques. Advantages of this choice are the low cost of the primary optical element, the absence of chromatic aberration in opposite to the standard Fresnel-lens solution, and the access to a solid know-how in the field of injection moulding processes.

\section{Mature technologies}

During the design process different solutions had been evaluated. Materials and production processes are tightly related to the final product characteristics, which are its efficiency and its cost.

Having this in mind, the TwinFocus concentrator design had been founded on some mature technologies, which give us the opportunity to produce a reliable and cost effective CPV system.

\footnotetext{
${ }^{a}$ Corresponding author: alessandro.sacca@unipd.it
}

This is an Open Access article distributed under the terms of the Creative Commons Attribution License 4.0, which permits unrestricted use, distribution, and reproduction in any medium, provided the original work is properly cited. 
- Triple-junction (3J) solar cells: multijunction solar cells are widely used in space applications since decades and they found their terrestrial applications since the mass production of the high efficiency triple-junction solar cells [3]. The choice of $3 \mathrm{~J}$ is currently the mainstream in CPV applications, and many research teams and private companies are moving around this topic.

- Injection moulding for plastic optical components: in these years important innovations interested the production of many optical components. An important innovation concerns the optical substrate, which could be plastic instead of glass in many applications. Important innovations can also be seen in the study of surface's geometry: improvements in numeric control machines give the opportunity to use freeform surfaces in place of conic ones [4]. Efforts in this field come from companies with interests in imaging as well as nonimaging applications.

- Thermal management: even though this seems a simple issue, a lot of companies and research groups are working in the study of the appropriate materials in order to reduce the thermal impedance between the electronic devices and their heat sinks, taking into account also other constraints such as dielectric strength.

\section{Key points in the design of a CPV module}

Before the presentation of the TwinFocus solution, we spend a few words in the description of the design process of a CPV module.

The three key concepts that a designer must consider and evaluate are: tracker's yearly energy production, reliability, and cost effectiveness. If a useful CPV solution exists, it must reach a trade-off between these key concepts.

Another important point is efficiency. The efficiency of a single CPV module has a many dependencies and it is fundamental to understand their priorities. Efficiency is affected by: cell's efficiency, optical efficiency, concentration factor, spectral content of the incoming radiation, cell's temperature, and illumination profile. This list is also a sort of ranking, from the most to the least important factor to consider. The design process usually begins with the definition of a merit function, and all these parameters must be included, each one with an appropriate weight associated.

Another fundamental aspect is the knowledge of the location where the CPV system will be placed. It is very important to have a Direct Normal Irradiance (DNI) database for that location, in order to predict the yearly energy production. Other important environmental information is the humidity level, the average air temperatures, the presence of sand, and the average wind speed and direction. Once the designer has as much information as possible, he or she can begin with the design of the CPV system.

\section{TwinFocus solution}

The basics of TwinFocus solution is represented in Fig. 1: the solar radiation passes through a front glass cover, is reflected by the Primary Optical Element (POE) onto a Secondary Optical Element (SOE) and then collected by a 3J solar cell. Each concentrator contains two solar cells and each half of the concentrator works as described above: this is the origin of the name TwinFocus.

Each concentrator has three main components, which are:

- the primary optical element: a plastic element made through an injection moulding process that is aluminized and protected against aging;

- the 3J solar cell soldered on a customized receiver;

- the heat sink: an aluminium element made through a die casting process. 

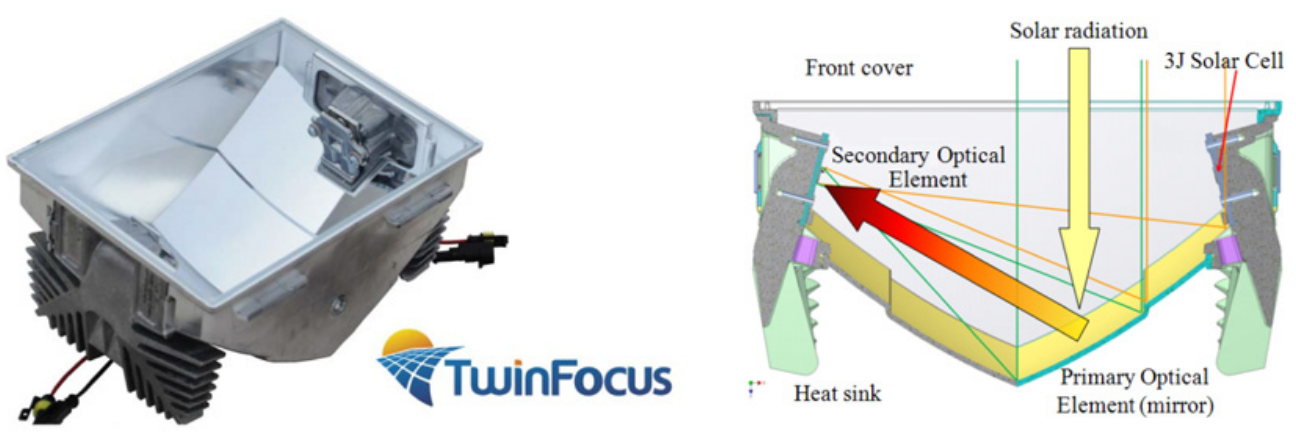

Figure 1. Left: picture of a complete TwinFocus concentrator. Right: schematic view of the main components inside the concentrator.

\subsection{Optics}

The optics is the heart of any CPV module. Efforts must be spent in designing an object that is optically efficient and that is ready for mass production at a suitable price. The main characteristics of a CPV optics are:

- the geometrical concentration factor, that is the ratio between the POE's active area and the 3J's area

- the acceptance angle, defined as the maximum tilt angle at which the number of collected rays is $90 \%$ of the rays collected by a perfectly aligned concentrator;

- the optical efficiency, that is the ratio between the number of rays hitting the front cover and the number of rays hitting the solar cell, also considering the radiation spectrum.

TwinFocus concentration factor is around $580 \mathrm{X}$ and this is due to the fact that the $3 \mathrm{Js}$ we use have their maximum efficiency close to that value.

The main feature of the optics is the POE, a faceted mirror with four sectors, which one reflects the sunlight directly on the $3 \mathrm{~J}$ cell. This solution, represented in Fig. 2, has been adopted in order to reduce the risks of "hot-spots" on the cell. Hot-spots are defined as solar cell's region with a concentration higher than 2000X. Figure 2 also represents the raytracing result, which is a uniform illumination profile with the desired concentration.

A SOE has also been designed in order to increase the acceptance angle, up to $1^{\circ}$ on both axes. The first TwinFocus release has a reflective SOE, but a refractive one has already been designed for the next release.

\subsection{Thermal management}

In TwinFocus configuration about $15 \mathrm{~W}$ of optical power are concentrated on an area that is $30.25 \mathrm{~mm}^{2}$. The problem is the management of such power, i.e. the cooling of the $3 \mathrm{~J}$ cell. The goals are then the reduction of the thermal resistance between the cell and the heat sink, and the study of the appropriate materials and shapes.

The design of the passive heat sink starts considering the worst case: high irradiation, no wind, high air temperature, bad tracker orientation, and disconnected electrical load. The final design was made iterating numerical simulations and experimental work.

Once the heat sink was studied and realized, the research was focused on the study of the small board on which the $3 \mathrm{~J}$ solar cell is soldered. Looking at Fig. 3, the goals are the study of the thermal impedance of different layers, and the dimensions of the receiver, in order to use it as a heat spreader. 

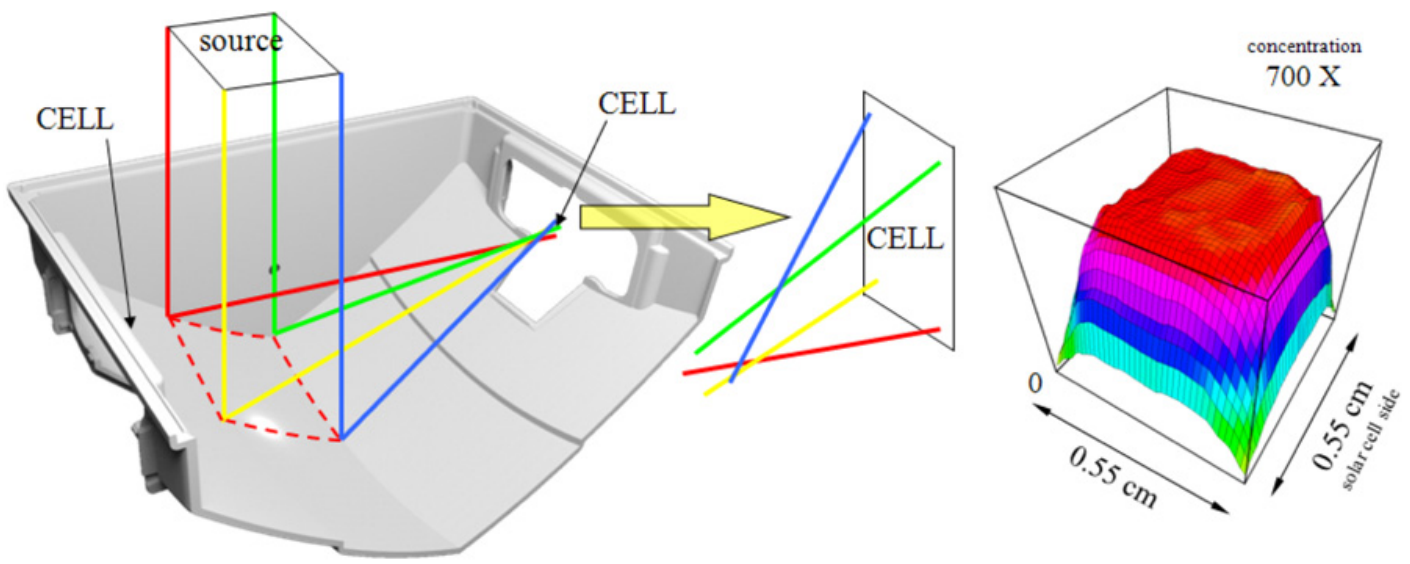

Figure 2. From the left: schematic view of the sunlight optical path, basic principle of the ray-tracing design, and the expected illumination profile of the $3 \mathrm{~J}$ solar cell.

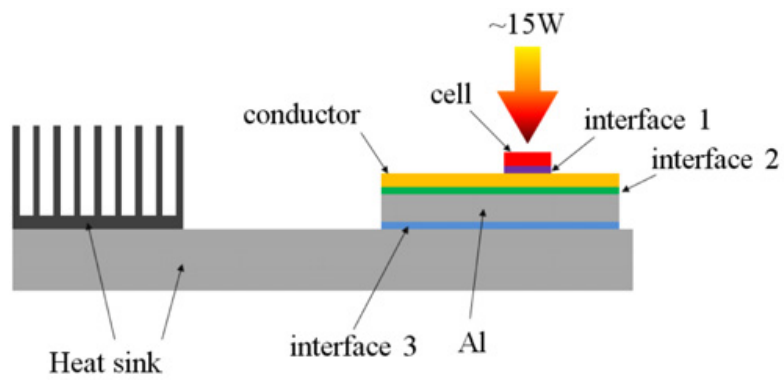

Figure 3. Thermal interfaces between the cell and the heat sink.

To achieve the first goal an original measurement set-up was realized for an in-sun flash tester. Using the cell's Open Circuit Voltage [5] as a meter of its temperature, an optical chopper was used to evaluate different materials and thickness: the shorter the thermal pulse, the closer to the cell is the tested layer.

\section{Outdoor measurements}

In Fig. 4 a complete TwinFocus tracker is shown. Four such trackers have already been installed in the north-east of Italy and are monitored since one year. A complete system is composed by a closed-loop dual axis tracking system, which is an altazimuthal mount, and 16 modules placed on two different planes with 40 concentrators each. The system peak power is $4.64 \mathrm{~kW}$ under Concentrator Standard Operating Conditions $^{\mathrm{b}}$, which means that the system has a peak efficiency of about $25 \%$.

Outdoor measurements of the whole system are measure of the maximum power point voltage and current after the inverter, the Direct Normal Irradiance, and the air temperature.

The graph in Fig. 4 represents the data of a typical sunny day of summer. The peculiar efficiency curve " $M$ " shape is due to the spectral changes during the day [6] and is typical of summer days in temperate zone's locations.

\footnotetext{
b CSOC: the conditions are $900 \mathrm{~W} / \mathrm{m}^{2}$ Direct Normal Irradiance with an AM1.5 spectral distribution, an air temperature of $20^{\circ} \mathrm{C}$, and a wind speed of $2 \mathrm{~m} / \mathrm{s}$.
} 

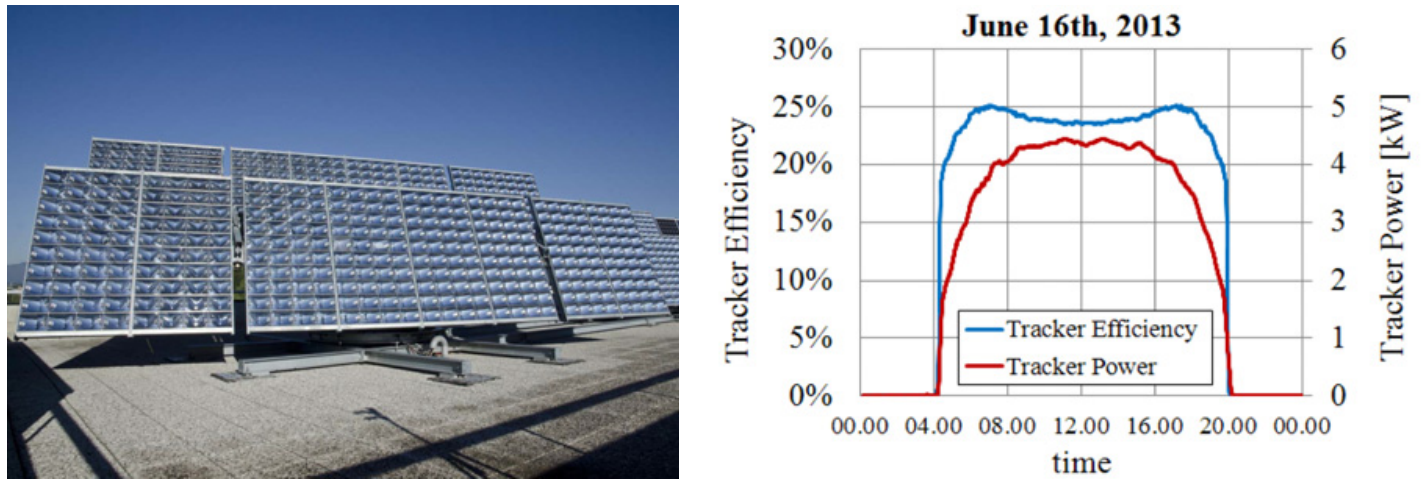

Figure 4. Left: photo of a complete TwinFocus tracker. Right: graph that represents the TwinFocus tracker performances during a summer sunny day.

\section{Expectations for the next release and Conclusions}

After one year of exposure to outdoor environment and work under operating conditions, we have some lessons learned.

The problems found on this short time-scale monitoring are basically about manufacturing complexity and reliability. The first one is almost typical of any prototype, and can be solved reaching an in-line production level. The second problem is about the moisture control inside the modules and the mirror aging. Reliability of a concentrator is one of the most challenging topics for any CPV producer, and the protection of optical, electrical, and mechanical components against environment for at least 20 years is not a mundane problem. For the second TwinFocus release some effort has been spent in the study of the appropriate protection of the mirror and the receiver. Attention has also been spent in the increase of the ratio between the optically active area and the gross area of a module. Other improvements in the second release are a higher concentration factor, which will be around 700X, a higher acceptance angle (up to $1.3^{\circ}$ ), a new receiver, and a different moisture management.

\section{References}

[1] P. Antonini, S. Centro, S. Golfetto, A. Saccà, SPIE Newsroom (2013) DOI: $10.1117 / 2.1201303 .004779$

[2] P. Antonini, S. Centro, S. Golfetto, A. Saccà, Il Nuovo Saggiatore 29 1-2, pp 39-50 (2013) http://prometeo.sif.it/papers/online/sag/029/01-02/pdf/06-fisica-e.pdf

[3] A. Luque and S. Hegedus, Handbook of Photovoltaic Science and Engineering, cap.9 (John Wiley \& Sons, 2003)

[4] J. Rolland, K. Thompson, SPIE Newsroom (2012) DOI: 10.1117/2.1201207.004309

[5] G.S. Kinsey et al, Prog. Photovolt: Res. Appl., 16:503-508 (2008)

[6] M. Muller, B. Marion, S. Kurtz, and J. Rodriguez, CPV-6 Conf., Freiburg, Germany (2010); http://www.nrel.gov/docs/fy11osti/47959.pdf 